\title{
Climatic-niche evolution with key morphological innovations across clades within Scutiger boulengeri (Anura: Megophryidae)
}

\author{
Xiuqin Lin ${ }^{1}$, Chungkun Shih ${ }^{2}$, Yinmeng $\mathrm{Hou}^{1}$, Xiaoxiao Shu ${ }^{1}$, Meihua Zhang ${ }^{1}$, Junhua \\ $\mathrm{Hu}^{1}$, Jiangping JIANG ${ }^{1}$, and Feng $\mathrm{Xie}^{1}$ \\ ${ }^{1}$ Chengdu Institute of Biology \\ ${ }^{2}$ Capital Normal University
}

March 9, 2021

\begin{abstract}
The studies of climatic-niche shifts over evolutionary time accompanied by key morphological innovations have attracted the interest of many researchers recently. We analyzed the realized niche dynamics across clades within Scutiger boulengeri using ecological niche models (ENMs), ordination method (environment principal component analysis; PCA-env), and correspondingly key morphological innovations combined phylogenetic comparative methods (PCMs) and phylogenetic generalized least squares (PGLS) regression methods throughout their distributions in Qinghai-Tibet Plateau (QTP) margins of China in Asia. Our analyses reveal that there is obvious niche divergence caused by niche expansion across $S$. boulengeri clades, especially in E. A, E. B and partial of E. C clades. Moreover, niche expansion is more popular than niche unfilling into novel environmental conditions. Annual mean temperature and Annual precipitation are the most important contributors in E. A and E. B clades, while Precipitation of driest month is most likely to be the leading limited factor in these two regions according to jackknife test of variable importance. In addition, we identified several key ecological and morphological traits that tend to be associated with niche expansion in S. boulengeri clades correspondingly. Specifically, we found that Elevation, Isothermality, Mean diurnal range and Max temperature of warmest month are significantly negative predictors of snout-vent length (SVL) under phylogenetic models, while the $S$. boulengeri toads from warmer and more arid environments tend to be larger. There seems to a trade-off strategy by trait covary of locomotor performance combined with enlarged SVL, which provide us a potential pattern of how a colonizing toad might seed a novel habit to begin the process of speciation and finally adaptive radiation. It is worth noting that we should not overlook that the Tibet continuously growing and moving northward over millions of years has laid the foundation for early divergence of clades within $S$. boulengeri.
\end{abstract}

\section{Introduction}

A major goal of ecology is in the inspection of niche evolution dynamics to explain rapid lineages diversity and mechanism of morphological evolution across clades, especially in complex mountainous regions. So far, most research efforts have focused on niche divergence and speciation, for example, several studies have found evidence for climatic niche conservatism among species (Crisp et al. 2009, Kozak and Wiens 2006, 2010), while others have shown evidence for niche divergence (Graham et al. 2004, Knouft et al. 2006, Evans et al. 2009, Hu et al. 2015). However, the potential effects and implicit meanings of intraspecific niche evolution dynamics across clades within species level are seldom known (Tingley et al. 2016).

In fact, niche of species or clades clearly do evolve, and niche shifts in range limits as a result of such evolution (Liu et al. 2020). Both ecological (available empty niches) and evolutionary changes (genetic drift or through selection) can potentially allow a species or clade to shift into a novel niche, and an observed shift can equally result from a change of the realized niche and the fundamental niche (Broennimann et al. 2007). Realized niche shifts can reflect ecological and evolutionary processes that lead to niche expansion or niche 
unfilling (Petitpierre et al. 2012, Strubbe et al. 2015). Specifically, niche expansion occurs when a species colonizes environmental conditions in its invaded range that are present, but unoccupied in its native range. Niche unfilling, another cause of realized niche shift, occurs when species fail to colonize climates in the invaded range that are occupied in the native range (Guisan et al. 2014), and this situation often reflects the fact that species have no sufficient time to colonize their potential range (Broennimann and Guisan 2008). A number of studies have reported that non-native shifted species can rapidly evolve to better adapt to various climatic pressures in the new niche range (Bonte and Saastamoinen 2012, Kooyers and Olsen 2012, Hudson et al. 2015). For instance, cane toads displayed an ability to colonize both highly arid and cold climates, one key mechanism for their colonization success is the up-regulation of genes associated with dispersal ability and metabolism (Rollins et al. 2015).

The Qinghai Tibetan Plateau (QTP) — the largest continental highland on Earth — is a major barrier to air flow in the atmosphere, which triggers the onset of the Indian summer monsoon (Molnar et al. 1993). Tibet continuously grew northward over millions of years in response to the thickening of Earth's crust associated with the collision of the Indian and Asian continental plates (Harrison et al. 1992), which is a long-standing topographic feature that arose from the collision between India and Asia (Rowley et al. 2006). The orogeny of high mountain ranges separating deep valleys might have created geographical barriers reducing gene flow between isolated populations and promoted allopatric divergence (Favre et al. 2015), in turn, the divergence time of clades species would have mapped the split time between two mountains. Meanwhile, novel environmental space released from biotic and abiotic constraints (Callaway and Maron 2006, Hierro et al. 2005), which provided key opportunities for occupation of novel niche especially in the early stages of clades divergence.

In addition, over some evolutionary time scale, niche evolution and ecological innovation have taken place (Peterson and Holt 2003). In some cases, niche evolution can be rapid and dramatic, as in adaptive radiations (Schluter 2000), a growing number of cases indicate the evolutionary shifts occurred in range limits with rapidly changing environments (Davis and Shaw 2001, Thomas et al. 2001, Evans et al. 2009). Moreover, prior researchers have documented morphological evolution is strongly influenced by ecological niche shifts in passerine birds (Alström et al. 2015), Eurasian perch (such as Perca fluviatilis, Bartels et al. 2012) and bivalved scallops (Sherratt et al. 2017). On the contrary, some studies have also found ecological radiation and morphological evolution can be largely de-coupled, both within and among species (Vanhooydonck and Van Damme 1999, Zaaf and Van Damme 2001). Eco-morphological studies vary in their overall scope, but there are relatively few studies that examine correlations between niche evolution and morphology variance within species level in amphibians, especially in toads. Neither the generality nor the possible adaptive significance of related traits versus climate relationships is clear.

Scutiger boulengeri toads have a wide range of distribution and occur along the eastern and southern slopes of the QTP at elevations between 2400 and $5270 \mathrm{~m}$ above sea level (Chen et al. 2009, Subba et al. 2015), which presents us an attractive system to study the role of niche evolution dynamics on phenotypic evolution. The capacity for rapid phenotypic evolution may directly facilitate species diversification by increasing the ability of a radiating clade to exploit ecological opportunities (Parent and Crespi 2009). However, to cope with changing ambient conditions on a shorter time-scale, ectotherms rely primarily on phenotypic plasticity (Angilletta 2009, Seebacher and Franklin 2011). In essence, from time to time, vacant niches are likely to be occupied by species that are already reasonably well adapted to them and are thereby able to produce viable populations that out-compete other invaders (Harvey and Rambaut 2000).

Herein, we use a set of climatic and morphological data and perform a synthesis of studies for assessing niche and morphological dynamics across clades within $S$. boulengeri. Specifically, we focus to address four key issues: (1) Are there niche divergence caused by niche shifts across clades? (2) Is such a divergence caused by niche unfilling or niche expansion? (3) Which climate variables contribute most to such a niche evolution dynamics? (4) Is there related trait evolution accompanied shifted niche when controlling for phylogenetic relatedness? We hypothesize that genetically isolated $S$. boulengeri clades will exhibit clearly segregated niche patterns and correspondingly morphological variation in this system. Specifically, we expect lower 
climatic niche overlap in shifted clades and observed values statistically significantly less than expected in bi-directions background test. Besides, clades with niche shifts owning trait coevolution for new life strategies combined for adapting novel niche.

\section{Material and methods}

\section{Phylogenetic Analysis}

Based on previous studies (Li et al. 2009, Hofmann et al. 2017), we identified Scutiger boulengeri containing six clades, for the first time, after max range field works. To construct a phylogeny for $S$. boulengeri clades, we used MEGA-X (Kumar et al. 2018) to align six selected mtDNA genomes (Genbank MW600725-MW600729 and FJ463132) with genome from outgroup Oreolalax omeimontis (Genbank EU180928). Phylogenetic trees were constructed separately by using maximum likelihood (ML) and Bayesian inference (BI) methods, both of which were implemented in PhyloSuite v1.2.1 (Zhang et al. 2020). The best-fit BIC substitution model (TPM2 + F + G4) was selected in ModelFinder (Kalyaanamoorthy et al. 2017). Divergence time for the reconstructed trees was estimated with the Reltime ML method using MEGA-X (Kumar et al. 2018). Based on previous research, we choose the Most Recent Common Ancestor (MRCA) of Scutiger and Oreolalax(53 Ma) as calibration point (Hofmann et al. 2017).

\section{Occurrence and environmental data}

We obtained locality point data for Scutiger boulengeri from our own fieldwork and the published literatures (Li et al. 2009, Hofmann et al. 2017). Localities cover Himalayas, QTP, Hengduan Mountains, Min Mountains and adjacent Mountains (Figure 1). Our final dataset comprises 96 geo-referenced occurrence records: 42 for East represented by 4 clades ( 9 for E. A clade, 9 for E. B, 10 for E. C, 14 for E. D) and 54 for West represented by 2 clades ( 35 for W. a clade and 19 for W. b).

We compiled 19 environmental variables and elevation for each period from the WorldClim database (Hijmans et al. 2005a) with a resolution of 30 seconds ( $1 \mathrm{~km}$ ) for each environment layer (Hijmans et al. 2005b). We included the dissimilarity of the enhanced vegetation index variable, drawn from the Global Habitat Heterogeneity project (Tuanmu and Jetz 2015). Because strong colinearity between environmental variables could inflate model accuracy (Veloz 2009, Boria et al. 2014), we examined pairwise correlations among the 21 variables within each clade distribution. We reduced autocorrelation of input environmental data by removing highly correlated variables with the threshold of Pearson's correlation tests $|\mathrm{r}|>0.8$ (Dormann et al. 2013). Finally, we chose 8 variables with lower correlation for subsequent analyses.

\section{Model evaluation of ENMs}

We use ENMeval R package to facilitate increased strictness in the development of Maxent models (Muscarella et al. 2014). The Akaike Information Criterion corrected (AICc) for small sample sizes reflects both model goodness-of-fit and complexity. The model with the lowest ${ }^{A} I C c v a l u e\left(i . e .{ }^{A} I C c=A I C c-A I C_{\min }=\right.$ 0)isconsideredthebestmodeloutof thecurrentsuiteofmodels.Weexploredmodelswithregularizationmultiplier (RM)valuesrc

\section{Suitable habitat prediction}

We use a maximum entropy modeling algorithm implemented in the program Maxent v.3.4.1 (Phillips et al. 2006, Phillips and Dudik 2008) to predict suitable habitat. Maxent uses environmental variables from localities at which a species has been documented previously to build a predictive model of where else the clades may occur due to the presence of similar environmental conditions (Elith et al. 2011). To assess model performance, we calculated the average value of the area under the receiver operating characteristic curve (AUC) for training and test datasets (Swets 1988), AUC takes on values ranging from 0.5 (no better discrimination than random) to 1 (perfect discrimination).

\section{Niche overlap and null hypothesis test}

One key assumption for applying ENMs is that species niche changes very slowly across space and time (Warren et al. 2008). These tests are based on two similarity metrics (Warren's I and Schoener's D), 
we calculated these metrics in ENMTools v1.4.3, using 100 replicates to generate a pseudoreplicated null distribution (Warren et al. 2010). The null hypothesis of niche equivalency is rejected when empirical values are significantly less than the critical values for both the niche equivalency and similarity tests (Warren et al. 2010).

E-space climate niche overlap between lineages of $S$. boulengeriwas estimated using the PCA-env approach proposed by Broennimann et al. (2012). An unbiased estimate of the Schoener's D metric can be calculated for our data and is ensured to be independent of the resolution of the grid, statistical confidence in niche overlaps was then tested through a bi-directions niche similarity test (Broennimann et al. 2012).

\section{Niche expansion and unfilling}

Schoener's D on species occupancy disentangles climate availability and the extent of niche divergence of clade pairs, but does not take into account the difference between partial filling and expansion (Petitpierre et al. 2012). However, expansions measured can characterize true niche shifts, when native and invaded ranges overlapped in climatic space, We followed Petitpierre et al. (2012), three categories were considered in: (1) stable environments where species occurs in both ranges, (2) unfilled environments where species occur only in the native range, and (3) expansion environments where the species occur only in the invaded range.

\section{Niche evolution}

We use phytools R package (Revell 2013) to visualize niche evolution throughout the phylogeny. We calculate the $\mathrm{K}$ value for a given trait and phylogeny, phytools package provides a randomization test to assess the significance of the observed K value (Revell et al. 2020). Finally, to assess the evolution mode along each climatic niche component (Cooper et al. 2010), we fit four alternative models of evolution for values of each climatic principal components (PCs): (a) Brownian motion model (BM) (Felsenstein 1985); (b) "Singlepeak" (OU) (Hansen 1997, Butler and King 2004); (c) an early-burst model (EB) (Harmon et al. 2010) and (d) a white noise model (WN). Calculations were conducted using the geiger package (Harmon et al. 2007), and the best-fitting model was chosen using the Akaike information criterion corrected (AICc) for small sample sizes and Akaike weights ( $\omega$ ) (Wagenmakers et al. 2004).

\section{Morphological data}

These data are derived from 151 specimens from our recent fieldworks and Herpetological Museum of the Chengdu Institute of Biology, CAS. The sample size varied between 8 and 42 specimens per clade (only male adults included), with a mean of 25 individuals per clade. The morphological variables including: snout-vent length (SVL), head length (HL), head width (HW), snout length (SL); inter-nasal space (INS), width of upper eyelid (UEW), inter-orbital space (IOS), diameter of eye (ED), length of lower arm and hand (LAHL), diameter of lower arm (LAD), hind-limb length (HLL), tibia length (TL), tibia width (TW), length of foot and tarsus (TFL) and foot length (FL), named system of morphological variables following Fei et al. (2005). Males within S. boulengeri predominant outstandingly in two pairs of keratinized spine patches on the chests and fingers of males and in variation adult body size and diameter of lower arm between them (Fu et al. 2007, Chen et al. 2009). In data analysis, we removed females for their insufficient quantity. Prior to all statistical analyses, the variables were log-transformed to better meet the requirements of normality and homogeneity (Rabosky and Adams 2012).

\section{PCMs and trait correlative analyses}

Biologists have long recognized that closely related species are generally more similar to one another than they are to more distantly related, which is often termed phylogenetic conservatism (Martin and Hansen 1997). Phylogenetic signal can be thought of as the degree to which similarity in trait values between species can be predicted upon their relatedness (Harvey and Rambaut 2000). If there is phylogenetic signal in the data, then PCMs are necessary for robust statistical analyses of trait correlations. To address the relationships between morphology and climate variables caused by niche evolution, we used PGLS (Grafen 1989, Martins and Hansen 1997). 


\section{Results \\ Phylogeny}

Due to the consensus trees of the Maximum Likelihood (ML) and Bayesian Inference (BI), we present only the ML tree for mtDNA gene cytochrome b (cytb) gene of the S. boulengeri clades in Figure 2a. The tree has high reconstruction confidence as the supporting values of the internal nodes are very high.

Molecular dating supports the earliest clade divergence of W. b (from Himalayas and QTP) to W. a (from the Hengduan Mountains) during the Miocene (13.72 Ma). Nearly at the same period around 13.39 Ma, W. a clade split from E. D, but, the remained clades gradually diverged around ca. 4-8 Ma.

\section{Environmental Data}

According to our Principal Component Analysis (PCA) of climate factors, the first five principal components explain $91.14 \%$ of variations (Table 1). PC1 is mainly represented by the increased temperature and reduced elevation, while PC2 explains increased precipitation, PC3 explains Mean diurnal range and Precipitation seasonality, both PC4 and PC5 represent extreme temperature and precipitation. The first three principal components are presented in Figure 3a, for more details, please see Table 1.

\section{Prediction of the Maxent distribution}

We choose the best model and find the corresponding RM and FCs parameters for Maxent model to facilitate increased rigour in the development of Maxent models (Table 2). Finally, the distributions based on Maxent across $S$. boulengeri clades were characterized by high AUC statistics, indicating that these ENMs successfully discriminated real occurrences from background locations. Jackknife tests on variable importance for $S$. boulengeri clades revealed that Precipitation of driest month in E. A, E. B and W. a clades, while Annual precipitation in E. C and E. D and Annual mean temperature in W. b produced the greatest decrease in gain when excluded from the model, suggesting these climate factors limit distributions of clades correspondingly, which likely to be the most important reasons for the next step niche divergence.

\section{Hypothesis tests based on ENMs and PCA-env approaches}

ENMs based niche equivalency tests reveal that 4 paired comparisons do not reject null distributions, seem equivalent as the values of observed niche overlap fall well in the middle of the null distributions (Supplementary material Appendix Figure A1). ENMs based background similarity tests indicate greater niche divergence between pairwise comparisons (2/15): E. B vs. W. b and E. A vs. E. B (Supplementary material Appendix Figure A2a, A2i). Interestingly, some pairwise comparisons (7/15) just show one-side significant divergence. The last part (6/15) niche overlap falls within the $95 \%$ confidence limits of the null distributions, leading to non-rejection of the hypothesis of retained niche conservatism.

The results of PCA-env based niche equivalency test (Figure 3d, 3e and Supplementary material Appendix Figure A3) show that pairwise comparisons of $80 \%(12 / 15)$ less than expected null distribution ranges and reject null hypotheses, indicating closely related clades are not equivalent to most related clades, and most clades have undergone significant alteration of their environmental niche and clades may be more resilient to climate changes than their native ranges suggest. Niche background test indicates that 11 paired comparisons show a very limited niche overlapping values (scores $<0.3$ ). Only 4 paired comparisons show niche overlap categorized as a moderate overlap (0.307-0.527). On the other hand, the background similarity tests indicate generally little niche similarity among the pairwise comparisons in six clades (Table 3 and Supplementary material Appendix Figure A4). The ordination null tests of niche similarity show that niches are less similar than random expectations in 30\% (5 paired comparison cases) in reciprocal directions, while 26\% (4 paired comparisons) are significantly not to reject the null hypothesis of niche conservatism in bi-directions.

\section{Niche expansion and unfilling dynamics}

As predicted, examining patterns of niche expansion and niche unfilling demonstrate a gradient of realized niche change across clades in their shifted ranges (Supplementary material Appendix Figure A5). There is 
considerable evidence of expansion when comparing the realized niche of clade to its native niche, such as, E. A clade shows $99.7 \%$ niche expansion (vs. native clade E. D) (Figure 3h). From our results (Table 3), there is nearly $50 \%$ (3/6; clade E. A, E. B and E. C) of the clades' invaded niche exists in climates that are less occupied in its native range (i.e. niche expansion). While, $33 \%$ (2/6; clade E. D and W. a) of the clades' native niches remain stable, only one clade W. b can be viewed as typical niche unfilling (Figure 3i).

\section{Niche evolution}

The results of phylogenetic signal test based on Blomberg's $\mathrm{K}$ show values in PC1 and PC2 are less than 1, best-fitted to WN model, which suggests that PC1 and PC2 dimension climate changes independence of phylogenetic relationships (data with no covariance structure among clades); but more than 1 in PC3, PC4 and PC5 indicate PC3, PC4 and PC5 dimension changes have closely related phylogenetic relationships (Table 4). The comparisons of model fit based on the AICc value and AICc weights $(\omega)$ indicate that the WN model is preferred in PC1 and PC2, where climatic components change in PC1 and PC2 regardless of shared ancestry between clades. Although niche conservatism is maintained in some clades for PC1 and PC2 (Figure 2c and Table 4), our tests of PC3, PC4 and PC5 fitted to a BM model, suggesting that following a divergence event along these climate PCs, clades branches may be subject to these variable environmental conditions, such as promoting the evolution of different climatic tolerances, which may be accumulated independently from ancestral ones.

\section{Morphological evolution}

The Blomberg's $K$ based test of phylogenetic signal analysis shows high value $(K>1)$ in morphological trait in SVL, SL and INS indicating relatively more phylogenetic signal. The comparisons of model fit based on the AICc and AICc weights $(\omega)$ indicate the BM model is preferred in SVL, SL and INS, where trait changes between clades can be predicted upon their relatedness. Following a divergence event along phylogeny, that is less labile than expected under a BM model of evolution clades branches may be subject to phylogeny, such as promoting the evolution of different morphological traits under climatic tolerances, which may be accumulated independently from ancestral ones. While, the others fitted a WN model, which seems to be unrelated with shared ancestry but preference of an adaptively phenotypic plasticity (Table 5), which may be closely related to habitat types adapted by clades.

\section{PCMs and trait correlative analyses}

According to PGLS, SVL and LAD are strongly related with variables of PC2 (dominated by precipitation) + PC3 (Mean diurnal range and Precipitation seasonality) + PC4 and PC5 (Min temperature of coldest month). Specifically, our results indicated that the evolution of the climate niche is associated with higher precipitation levels and Min temperature of coldest month, both of which conditions are principally presented in the distribution in E. A and E. B clades, which suggested SVL and LAD may be controlled by Mean diurnal range, precipitation and extreme temperature. In the same vein, HW and SL are controlled by $\mathrm{PC} 1+\mathrm{PC} 3+\mathrm{PC} 4+\mathrm{PC} 5(\mathrm{P}<0.05)$, which means temperature contributes most in these traits. $\mathrm{PC} 1+\mathrm{PC} 2+\mathrm{PC} 3+\mathrm{PC} 5$ best fitted in $\mathrm{ED}(\mathrm{P}<0.05)$ and TW controlled by $\mathrm{PC} 1+\mathrm{PC} 2+\mathrm{PC} 3+\mathrm{PC} 4(\mathrm{P}<0.05)$. Interestingly, UEW significant to $\mathrm{PC} 1(\mathrm{P}<0.05)$ but selected model by $\mathrm{AICc}$ is $\mathrm{PC} 1+\mathrm{PC} 2+\mathrm{PC} 3+\mathrm{PC} 4(\mathrm{P}$ $>0.05)$. For other best fitted models, please see Table 6 .

\section{Discussion}

As documented in Guisan et al. (2014) and Sexton et al. (2017), recent applications of niche models (based on ENMs and PCA-env) analyze niche shifts have provided new insights to the roles of abiotic climate and geographical conditions in shaping range limits. Moreover, intraspecific variation along allopatric genetic clades in the potential for realized niche shifts has important implications not only for forecasting biological invasion, but for studying the process of origin and evolution across clades within species level.

Our results agree with previous progressive uplifts of Tibet (Mulch et al. 2006). Molecular datings of E. D, $\mathrm{W}$. a and W. b are almost identical to the fourth uplift of high mountain ranges and aridification of Central Asia (ca. 20 to $10 \mathrm{Ma}$ ), and the divergence times of clades E. A, E. B and E. C are coincident with the final 
extension of the last uplift (ca. 10 Ma to present) predicted by previous studies (Favre et al. 2015). However, future researches with more detailed complementary analyses are needed to understand a link among clades molecular dating, gene flow routes and past geological and climatic changes across these regions.

Our results show PCA-env based approach well supports E. A, E. B and partial of E.C clades with significant divergence. However, ENMs based method just supports divergence of E. B in bi-directions, while the divergence of E. A exhibits one-sided significance, the opposite comparisons not deviated from null expectation. While inconsistent conclusions drawn in divergence of $\mathrm{E}$. C based two methods. In literatures, inconsistent conclusions regarding niche conservatism or divergence have been frequently reported based on different approaches. For example, Guo et al. (2013) applied the ordination and ENMs for the globally introduced Phragmites australis and found inconsistent results. Our results based on two approaches draw almost the same conclusion in one-sided tests, but PCA-env based approach is sensitive to niche divergence, while ENMs is more inclined to niche conservatism in bi-directions. We analyze reasons leading to these differences further and find the native-range model, such as ENMs, always under-predicts the species' invaded range in exactly the types of climates in which there was evidence of niche expansion as shown in previous studies. Strubbe et al. (2013) showed that the predictive performance of native range ENMs increased with increasing niche overlap and decreased with increasing niche change. Similarly, Tingley et al. (2014) found that a native-range ENMs under-predicted the extent of the species' Australian invasion.

In addition, we found E. A, E. B and E. C clades within S. boulengeri have an obviously expansion of climatic niche (Figure 3h and Supplementary material Appendix Figure A5). Intriguingly, they shift beyond the realized niche with the new conditions but still overlap the fundamental niche, which provides positive proof for the niche conservatism hypothesis (Wiens 2004, Kozak and Wiens 2006) and niche divergence hypothesis (Graham et al. 2004, Evans et al. 2009) are not contradictory. We found a gradient of realized niche change in the invaded ranges across clades within $S$. boulengeri : niche stasis on E. D (96.3\%) and W. a (96.6\%), niche unfilling in W. b (75.2\%), and niche expansion (vs. E. D and W. a separately) in E. A (mean $=99.3 \%)$, E. B $($ mean $=97.1 \%)$ and E. C (mean $=90 \%)$. Our results seem to be inconsistent with conclusion of previous studies in Petitpierre et al. (2012), in which realized niche shifts between the native and invaded ranges were largely due to niche unfilling. Our results are also different from the results of cane toad in Tingley et al. (2014): the shift in the realized niche of the cane toad Rhinella marina was solely due to niche expansion. In our results, niche expansion into novel environments is more popular than niche unfilling, suggesting that our niche divergence due to niche expansion in the shifted range and thus represents true niche changes. Why do E. D and W. a fail to fill its fundamental niche in its native range? One possibility is that the presence of closely related species (S. glandulatus and/or S. mammatus ) may prevent S. boulengeri from colonizing suitable environments south of its present range. Indeed, previous study found there were low rates of interspecific hybridization (Chen et al. 2009). We cannot exclude dispersal limitation in the native range as a possible contributing factor, such as Jinsha River and Yalong River (Li et al. 2009), which also can enforce stable parapatric range boundaries. Future studies will be able to test this hypothesis using laboratory or field experiments.

Numerous examples of rapid adaptation in non-native niche suggest that rapid evolution may be common during invasions in species level (Alström et al. 2015, Bartels et al. 2012, Sherratt et al. 2017). The degree to which species adapt to novel environments is important to a range of topics in ecology and evolution (Wiens et al. 2008), but is of special concern for the study of intraspecific niche evolution (Tingley et al. 2016). In our study, niche divergence caused by niche expansion indeed accompanied key morphological innovations of preadaption in novel climates vs. niche unfilling and stability. Our finding of significant phylogenetic signal in SVL (Table 5) and Elevation (Table 4 and Figure 2c), indicates that these acquired data are not random and our results are robust. Furthermore, our findings of significant phylogenetic signal in these traits are consistent with previous studies (Freckleton et al. 2002, Blomberg et al. 2003, Oufiero et al. 2011). We found that Elevation ( $\mathrm{AIC}=21.3 ; \mathrm{P}=0.002)$, Isothermality $(\mathrm{AIC}=24.47 ; \mathrm{P}=0.007)$, Mean diurnal range (AIC $=29.33 ; \mathrm{P}=0.037)$ and Max temperature of warmest month $(\mathrm{AIC}=29.31 ; \mathrm{P}=0.037)$ are significantly negative predictors of SVL under phylogenetic models, which suggest $S$. boulengeri toads from warmer and more arid environments tend to be larger, which is in concert with true records in our field work. 
Several factors may underlie the observed pattern of SVL variation inS. boulengeri clades. One possibility pertains to the expected relationship between fasting endurance and SVL (Mautz 1982). The second possibility is ecological release in novel shifted areas may allow for larger SVL (Losos and Queiroz 1997, Yoder et al. 2010). The third is likely that maintenance of preferred body temperature influences the evolution of SVL (Oufiero et al. 2011). Our results highlight reduced competitors (ecological release) in newly shifted niche may be the most likely reason for enlarged SVL in E. A, E. B and E. C clades.

Moreover, we found species tolerance of newly shifted niche tends to have morphological attributes important for locomotor performance. These traits may be a key preadaptation in toads that helps overcoming the challenge of insufficient precipitation or high temperature in novel habitats, which are in accord with the evolutionary shifts mechanistic model highlighted by prior studies (Tingley et al. 2014, Phillips et al. 2010, Kolbe et al. 2010). In our study, LAHL (mean value: $27.19 \mathrm{~mm}$ in clade E. A, $26.27 \mathrm{~mm}$ in clade E. B and $23.95 \mathrm{~mm}$ in E. C, $21.77-23.57 \mathrm{~mm}$ in the remained clades, Supplementary material Appendix Table A1), HLL and FL have the same trend. Interestingly, these character values, without size-correction, have a high phylogenetic signal, best fitted BM model. However, once size-corrected, these character values will have a completely different scenario - with a low phylogenetic signal but trait correlations still exist, there seems to be a trade-off strategy by locomotor performance combined enlarged SVL for speed and endurance in thermal reaction norms (Angilletta et al. 2003). Collectively, we found phenotypic plasticity (i.e LAHL, HLL and FL) and evolution changes (i.e. SVL) may together contribute to niche expansion towards adapting novel niche. Indeed, because the proximate mechanisms that underlie variation between body length and locomotor performance can be complex, quantifying the fitness consequences of the resulting trade-offs will be challenging, novel analytical tools and optimization models are needed in further studies.

\section{Conclusion}

Combing distinct genetic clades from different geographic areas with correlative niche models and morphological evolution models, we have shown considerable variations in the degree of realized niche expansion and unfilling across the clades within $S$. boulengeri toads in the Qinghai-Tibet Plateau region. In the case of $S$. boulengeri toads, niche divergence occurs accompanied by niche expansion rather than unfilling, niche expansion are more prevalent than niche unfilling in E. A, E. B and E. C clades, while niche unfilling presents just in W. b clade.

Meanwhile, niche divergence caused by niche expansion indeed accompanies key morphological innovations of preadaption in novel climates than niche unfilling and stability. Factors such as enlarged body size and enhanced locomotor performance have been shown to increase expansion success by helping toads to cope with novel conditions.

Recognizing true niche shifts accompanied by key morphological innovations do exist, further assessments should seek to understand molecular mechanisms of key morphological innovations or related life strategies combined that have allowed these particular clades to expand their niches dramatically. It would be particularly interesting to use the same framework to test whether the same pattern is found in other organisms, especially in such widespread species or clades in future.

\section{References}

Alström, P. et al. 2015. Dramatic niche shifts and morphological change in two insular bird species. - R. Soc. Open Sci. 2: 140364.

Angilletta, M. J. et al. 2003. Tradeoffs and the evolution of thermal reaction norms. - Trends Ecol. Evol. 18: 234-240.

Angilletta, M. J. 2009. Thermal Adaptation: A Theoretical and Empirical Synthesis. - Oxford University Press.

Bartels, P. et al. 2012. Water Transparency Drives Intra-Population Divergence in Eurasian Perch (Perca fluviatilis). - PLoS ONE 7 (8): e43641. Blomberg, S. P. et al. 2003. Testing for phylogenetic signal in 
comparative data: Behavioral traits are more labile. - Evolution 57: 717-745. Bonte, D. and Saastamoinen, M. A. K. 2012. Dispersal syndromes in butterflies and spiders. - In: Clobert, J. et al. (ed.), Dispersal Ecology and Evolution, Oxford University Press, pp. 161-170.

Boria, R. A. et al. 2014. Spatial filtering to reduce sampling bias can improve the performance of ecological niche models. - Ecol. Model. 275: 73-77.

Broennimann, O. and Guisan, A. 2008. Predicting current and future biological invasions: Both native and invaded ranges matter. - Biol. Lett. 4: 585-589.

Broennimann, O. et al. 2007. Evidence of climatic niche shift during biological invasion. - Ecol. Lett. 10: 701-709.

Broennimann, O. et al. 2012. Measuring ecological niche overlap from occurrence and spatial environmental data. - Global Ecol. Blogeogr. 21 (4): 481-497. Butler, M. A. and King, A. A. 2004. Phylogenetic comparative analysis: A modeling approach for adaptive evolution. - Am. Nat. 164 (6): 683-695.

Callaway, R. M. and Maron, J. L. 2006. What have exotic plant invasions taught us over the past 20 years? - Trends Ecol. Evol. 21: 369-374.

Chen, W. et al. 2009. Frequent Mitochondrial Gene Introgression among High Elevation Tibetan Megophryid Frogs Revealed by Conflicting Genegenealogies. - Mol. Ecol. 18: 2856-2876.

Cooper, N. W. et al. 2010. Reproductive correlates of spring arrival date in the eastern kingbird Tyrannus tyrannus. - J. Ornithol. 152: 143-152.

Crisp, M. D. et al. 2009. Phylogenetic biome conservatism on a global scale. - Nature 458: 754-758.

Davis, M. B. and Shaw, R. G. 2001. Range shifts and adaptive responses to quaternary climate change. Science 292: 673-679.

Dormann, C. F. et al. 2013. Collinearity: a review of methods to deal with it and a simulation study evaluating their performance. - Ecography 36, 27-46.

Elith, J. et al. 2011. A statistical explanation of MaxEnt for ecologists. - Divers. Distrib. 17 (1): 43-57.

Evans, M. E. K. et al. 2009. Climate, Niche Evolution, and Diversification of the "Bird-Cage" Evening Primroses (Oenothera, Sections Anogra and Kleinia). - Am. Nat. 173: 225-240.

Favre, A. et al. 2015. The role of the uplift of the Qinghai-Tibetan Plateau for the evolution of Tibetan biotas. - Biol. Rev. 90: 236-253.

Fei, L. et al. 2005. An illustrated Key to Chinese Amphibians. - Chengdu: Sichuan Publishing House of Science and Technology.

Felsenstein, J. 1985. Confidence limits on phylogenies: an approach using the bootstrap. - Evolution: 783-791.

Freckleton, R. et al. 2002. Phylogenetic analysis and comparative data: a test and review of evidence. - Am. Nat. 160: 712-726. Fu, J. Z. et al. 2007. A phylogeny of the high-elevation Tibetan megophryid frogs and evidence for the multiple origins of reversed sexual size dimorphism. - J. Zool. 273: 315-325. Grafen, A. 1989. The phylogenetic regression. - Philos. Trans. R. Soc. Lond. B Biol. Sci. 326: 119-157.

Graham, C. H. et al. 2004. Integrating phylogenetics and environmental niche models to explore speciation mechanisms in dendrobatid frogs. - Evolution 58: 1781-1793.

Guisan, A. et al. 2014. Unifying niche shift studies: Insights from biological invasions. - Trends Ecol. Evol. 29: 260-269.

Guo, W. Y. et al. 2013. Invasion of Old World Phragmites australis in the New World: Precipitation and temperature patterns combined with human influences redesign the invasive niche. - Glob. Change Biol. 19: $3406-3422$. 
Hansen, T. F. 1997. Stabilizing selection and the comparative analysis of adaptation. -Evolution 51 (5): 1341-1351. Harmon, L. J. et al. 2007. GEIGER: Investigating evolutionary radiations. - Bioinfor- matics 24 (1): 129-131. Harmon, L. J. et al. 2010. Early bursts of body size and shape evolution are rare in comparative data. - Evolution 64 (8): 2385-2396.

Harrison, T. M. et al. 1992. Raising Tibet. - Science 255: 1663-1670.

Harvey, P. H. and Rambaut, A. 2000. Comparative analyses for adaptive radiations. - Philos. Trans. R. Soc. Lond. B. Biol. Sci. 355: 1599-1605.

Hierro, J. L. et al. 2005. A biogeographical approach to plant invasions: The importance of studying exotics in their introduced and native range. - J. Ecol. 93: 5-15.

Hijmans, R. J. et al. 2005a. WorldClim, Version 2.1. $-<$ https://www.worldclim.org /data/worldclim21.html>.

Hijmans, R. J. et al. 2005b. Very high resolution interpolated climate surfaces for global land areas. - Int. J. Climatol. 25: 1965-1978.

Hofmann, S. et al. 2017. Molecular Phylogenies Indicate a Paleo-Tibetan Origin of Himalayan Lazy Toads (Scutiger ). - Sci. Rep. 7: 3308.

Hu, J. H. et al. 2015. Niche divergence accelerates evolution in Asian endemic Procapra gazelles. - Sci. Rep. 5: 10069.

Hudson, C. M. et al. 2015. Virgins in the vanguard: low reproductive frequency in invasion front cane toads. - Biol. J. Linnean Soc. 116: 743-747.

Kalyaanamoorthy, et al. 2017. ModelFinder: fast model selection for accurate phylogenetic estimates. - Nat. Methods 14: 587-589.

Knouft, J. H. et al. 2006. Phylogenetic analysis of the evolution of the niche in lizards of the Anolis sagrei group. - Ecology 87: 29-38.

Kolbe, J. J. et al. 2010. Modeling the consequences of thermal trait variation for the cane toad invasion of Australia. - Ecol. Appl. 20 (8): 2273-2285.

Kooyers, N. J. and Olsen K. M. 2012. Rapid evolution of an adaptive cyanogenesis cline in introduced North American white clover (Trifolium repens L. ). - Mol. Ecol. 21: 2455-2468.

Kozak, K. H. and Wiens, J. J. 2010. Accelerated rates of climatic-niche evolution underlie rapid species diversification. - Ecol. Lett. 13: 1378-1389.

Kozak, K. H. and Wiens, J. J. 2006. Does niche conservatism promote speciation? A case study in North American salamanders. - Evolution 60: 2604-2621.

Kumar, S. et al. 2018. MEGA X: Molecular Evolutionary Genetics Analysis across Computing Platforms. Mol. Biol. Evol. 35 (6): 1547-1549.

Li, R. 2009. Rivers as barriers for high elevation amphibians: a phylogeographic analysis of the alpine stream frog of the Hengduan Mountains, - J. Zool. 277 (4): 309-316.

Liu, C. L. et al. 2020. Most invasive species largely conserve their climatic niche. - Proc. Natl Acad. Sci. USA 117: 23643-23651.

Losos, J. B. and Queiroz, K. D. 1997. Evolutionary consequences of ecological release in Caribbean Anolis lizards. - Biol. J. Linnean Soc. 61: 459-483.

Martins, E. P. and Hansen, T. F. 1997. Phylogenies and the comparative method: a general approach to incorporating phylogenetic information into the analysis of interspecific data. - Am. Nat. 149: 646-667. 
Mautz, W. J. 1982. Patterns of evaporative water loss. - In Gans, C. and Pough, F. H. (ed.), Biology of the reptilian. Academic Press, pp. 443-481. Molnar, P. et al. 1993. Mantle dynamics, uplift of the Tibetan Plateau and the Indian monsoon. - Rev. Geophys. 31: 357-96.

Mulch, A. and Chamberlain, C. P. 2006. Earth science - The rise and growth of Tibet. - Nature 439: 670-671.

Muscarella, R. et al. 2014. ENMeval: an R package for conducting spatially independent evaluations and estimating optimal model complexity for Maxent ecological niche models. - Methods Ecol. Evol. 5: 11981205 .

Oufiero, et al. 2011. Swimming performance trade-offs across a gradient in community composition in Trinidadian killifish (Rivulus hartii ). - Ecology 92: 170-179.

Parent, C. E. and Crespi, B. J. 2009. Ecological opportunity in adaptive radiation of Galapagos endemic land snails. - Am. Nat. 174: 898-905.

Peterson, A. T. and Holt, R. D. 2003. Niche differentiation in Mexican birds: Using point occurrences to detect ecological innovation. - Ecol. Lett. 6: 774-782.

Petitpierre, B. et al. 2012. Climatic niche shifts are rare among terrestrial plant invaders. - Science 335: 1344-1348.

Phillips, B. L. et al. 2006. Invasion and the evolution of speed in toads. - Nature 439: 803.

Phillips, B. L. et al. 2010. Evolutionarily accelerated invasions: The rate of dispersal evolves upwards during the range advance of cane toads. - J. Evol. Biol. 23 (12): 2595-2601.

Phillips, S. J. and Dudík, M. 2008. Modeling of species distributions with Maxent: new extensions and a comprehensive evaluation. - Ecography 31: 161-175.

Rabosky, D. L. and Adams, D. C. 2012. Rates of morphological evolution are correlated with species richness in salamanders. - Evolution 66: 1807-1818.

Revell, L. J. 2013. Two new graphical methods for mapping trait evolution on phylogenies. - Methods Ecol. Evol. 4: 754-759. Revell, L. J. 2020. Package phytools, Version 0.7-70. - <https://github.com /liamrevell/phytools $>$.

Rollins, L. A. et al. 2015. Genetic perspective on rapid evolution in cane toads (Rhinella marina). - Mol. Ecol. 24: 2264-2276.

Rowley, D. and Currie, B. S. 2006. Palaeo-altimetry of the late Eocene to Miocene Lunpola basin, central Tibet. - Nature 439: 677-681.

Schluter, D. 2000. The ecology of adaptive radiation. - Oxford Univ. Press.

Seebacher, F. and Franklin, C. E. 2011. Physiology of invasion: cane toads are constrained by thermal effects on physiological mechanisms that support locomotor performance. - J. Exp. Biol. 214: 1437-1444.

Sexton, J. P. et al. 2017. Evolution of ecological niche breadth. - Annu. Rev. Ecol. Evol. Syst. 48: 183-206.

Sherratt, E. et al. 2017. Rates of morphological evolution, asymmetry and morphological integration of shell shape in scallops, - BMC Evol. Biol. 17: 248.

Strubbe, D. et al. 2015. Niche conservatism among non-native vertebrates in Europe and North America. Ecography 38: 321-329.

Strubbe, D. et al. 2013. Niche conservatism in non-native birds in Europe: niche unfilling rather than niche expansion. - Global Ecol. Biogeogr. 22: 962-970. 
Subba, B. et al. 2015. Scaling new heights: first record of Boulenger's Lazy Toad Scutiger boulengeri (Amphibia: Anura: Megophryidae) from high altitude lake in Sikkim Himalaya, India. - J. Threatened Taxa 7 (10): 7655-7663. Swets, J. 1988. Measuring the accuracy of diagnostic systems. - Science 240 (4857): 1285-1293.

Thomas, C. D. et al. 2001. Ecological and evolutionary processes at expanding range margins. - Nature 411: $577-581$.

Tingley, R. et al. 2014. Realized niche shift during a global biological invasion. - Proc. Natl Acad. Sci. USA 111: $10233-10238$.

Tingley, R. et al. 2016. Patterns of niche filling and expansion across the invaded ranges of an Australian lizard. - Ecography (Cop.) 39: 270-280.

Tuanmu, M. N. and Jetz, W. 2015. A global, remote sensing-based characterization of terrestrial habitat heterogeneity for biodiversity and ecosystem modelling. - Glob Ecol. Biogeogr. 24: 1329-1339.

Vanhooydonck, B. and Van Damme, R. 1999. Evolutionary relationships between body shape and habitat use in lacertid lizards. - Evol. Ecol. Res. 1: 785-805.

Veloz, S. D. 2009. Spatially autocorrelated sampling falsely inflates measures of accuracy for presenceonly niche models. - J. Biogeography 36: 2290-2299.

Wagenmakers, E. J. and Farrell, S. 2004. AIC Model Selection Using Akaike Weights. - Psychon. Bull. Rev. 11: $192-196$.

Warren, D. L. et al. 2010. ENMTools: a toolbox for comparative studies of environmental niche models. Ecography 33: 607-611.

Warren, et al. 2008. Environmental niche equivalency versus conservatism: quantitative approaches to niche evolution. - Evolution 62 (11): 2868-2883. Wiens, J. J. 2004. Speciation and Ecology Revisited: Phylogenetic Niche Conservatism and the Origin of Species. - Evolution 58 (1): 193-197.

Wiens, J. J. et al. 2008. Environmental niche equivalency versus conservatism: quantitative approaches to niche evolution. - Evolution 62: 2868-2883.

Yoder, J. B. et al. 2010. Ecological opportunity and the origin of adaptive radiations. - J. Evol. Biol. 23: 1581-1596.

Zaaf, A. and Van Damme, R. 2001. Limb proportions in climbing and ground-dwelling geckoes (Lepidosauria, Gekkonidae): a phylogenetically informed analysis. - Zoomorphology 121: 45-53.

Zhang, D. et al. 2020. PhyloSuite: an integrated and scalable desktop platform for streamlined molecular sequence data management and evolutionary phylogenetics studies. - Mol. Ecol. Resour. 20: 348-355.

Supplementary material Appendix at <www.oikosoffice.lu.se/appendix >.

Acknowledgements - We thank Chunlong Liu from Freie Universität Berlin \& Université Paris Saclay for help on conception of the earlier draft. We thank Dr. Cheng Li, Dr. Yin Qi, Dr. Guocheng Shu and Professor Dajie Gong, Shengchao Shi and Xiancheng Xu for their kind help in field work.

Funding - This study was funded by the Second Tibetan Plateau Scientific Expedition and Research Program (STEP, 2019QZKK05010503), the Biodiversity Survey and Assessment Project of the Ministry of Ecology and Environment, China 2019HJ2096001006, Construction of Basic Conditions Platform of Sichuan Science and Technology Department 2019JDPT0020 and China Biodiversity Observation Networks (Sino BON).

Author contributions - XQL and FX conceived the idea; XQL and FX collected the data with assistance from YMH, XXS, MHZ, JHH and JPJ; XQL and FX processed the raw data; XQL and CKS analyzed the data; XQL, CKS and FX leaded the writing. All authors contributed with edits and comments toward the final manuscript. 


\section{Data archiving statement}

WorldClim database is available at $<$ http://www.worldclim.org/ $>$. The Global Habitat Heterogeneity's raster layers are available at $<$ https://www.earth env.org $>$. The mtDNA genomes data are available at $<$ https://www.ncbi.nlm.nih.gov/>. All of the analysed datasets and R-scripts generated in our study are available from the first author upon request.

\section{Conflict of interest statement}

The authors declare no conflicts of interest.

\section{Ethics statement}

All animal handling and processing were in accordance with the Law of the People's Republic of China on the Protection of Wildlife and approved by the Animal Care Committee of CIB, Chinese Academy of Sciences.

\section{Figure and table captions}

Figure 1. Clades' distributions based on occurrence records for Scutiger boulengeri. Map showing the Tibet regions including main Mountains and main Rivers. Abbreviations: YTR: Yarlung-Tsangpo River; NR: Nu River; LCR: Lancang River; JSR: Jinsha River; YLR: Yalong River; DDR: Dadu River; MR: Min River; YTZR: Yangtze River; YR: Yellow River; Mts.: Mountains.

Figure 2. (a): Phylogenetic relationships and geographic distributions in Minimum convex polygon (MCP) and the suitable range of six clades in S. boulengeri ; supporting values of BI and ML labeled on branches; divergence time (Ma) of dated tree in bold; (b): The suitable habitat range prediction map for each clade; (c): Ancestral state reconstructions of main climatic PCs and Elevation, colors of branches reflect values of PCs and elevation interpolating the states along each edge.

Figure 3. (a): Three dimensional principal component analysis (3D-PCA) of climate variables across six clades withinS. boulengeri; (b): Predicted occupied niche of Isothermality across six clades within S. boulengeri ;(c): Correlation and contribution of each variable to the first two components of the PCA-env; (d)-(e): Histograms of niche equivalency distributions, diamond lines represent observed values; (f)-(g) Histograms of niche similarity distributions in bi-directions; (h)-(i): Two pairwise comparisons of niche dynamic between native and shifted ranges in environmental space depicted by the first two axes of a PCA, calibrated on the entire range of conditions available in China (red solid lines). Niche expansion, overlap and unfilling situations are stacked in the environmental space for each clade. Green areas represent climates only occupied in the native range and blue indicate climates occupied in both the native and non-native range, while red areas indicate niche expansion in the shifted range. Shading indicates the density of occurrences of the species by cells in the native range. The solid and dashed contour lines illustrate, respectively, $100 \%$ and $50 \%$ of the available environment in the native range.

Table 1 Loadings on the first five principal components.

\begin{tabular}{llllll}
\hline Variables & PC1 & PC2 & PC3 & PC4 & PC5 \\
\hline Annual mean temperature (bio1) & $\mathbf{0 . 9 3 5}$ & 0.271 & 0.037 & 0.219 & -0.033 \\
Mean diurnal range (bio2) & -0.187 & -0.173 & $\mathbf{0 . 8 1 9}$ & 0.153 & -0.199 \\
Isothermality (bio3) & -0.240 & 0.320 & $\mathbf{0 . 6 8 6}$ & 0.518 & -0.205 \\
Temperature seasonality (bio4) & 0.227 & -0.707 & -0.182 & -0.550 & 0.171 \\
Max temperature of warmest month (bio5) & $\mathbf{0 . 9 8 3}$ & -0.083 & 0.000 & 0.028 & -0.004 \\
Min temperature of coldest month (bio6) & 0.034 & -0.396 & 0.217 & $\mathbf{0 . 6 5 5}$ & $\mathbf{0 . 5 6 3}$ \\
Temperature annual range (bio7) & 0.121 & 0.383 & -0.218 & $\mathbf{- 0 . 6 5 1}$ & $\mathbf{- 0 . 5 6 5}$ \\
Mean temperature of wettest quarter (bio8) & 0.905 & 0.230 & 0.055 & -0.119 & 0.269 \\
Mean temperature of driest quarter (bio9) & 0.703 & 0.243 & 0.039 & 0.586 & -0.264 \\
Mean temperature of warmest quarter (bio10) & $\mathbf{0 . 9 9 4}$ & 0.046 & -0.035 & 0.050 & 0.018 \\
Mean temperature of coldest quarter (bio11) & 0.783 & 0.465 & 0.088 & 0.383 & -0.081
\end{tabular}




\begin{tabular}{llllll}
\hline Variables & PC1 & PC2 & PC3 & PC4 & PC5 \\
\hline Annual precipitation (bio12) & -0.173 & 0.872 & -0.348 & -0.137 & 0.051 \\
Precipitation of wettest month (bio13) & -0.176 & $\mathbf{0 . 9 3 9}$ & 0.191 & -0.033 & 0.135 \\
Precipitation of driest month (bio14) & -0.361 & 0.241 & -0.624 & 0.353 & 0.347 \\
Precipitation seasonality (bio15) & -0.012 & -0.080 & $\mathbf{0 . 8 7 9}$ & -0.176 & 0.266 \\
Precipitation of wettest quarter (bio16) & -0.213 & $\mathbf{0 . 9 3 5}$ & 0.127 & -0.124 & 0.141 \\
Precipitation of driest quarter (bio17) & -0.317 & 0.206 & -0.770 & 0.431 & 0.097 \\
Precipitation of warmest quarter (bio18) & -0.216 & $\mathbf{0 . 9 1 7}$ & 0.153 & -0.166 & 0.217 \\
Precipitation of coldest quarter (bio19) & -0.224 & -0.231 & -0.500 & $\mathbf{0 . 6 3 4}$ & $\mathbf{- 0 . 4 2 5}$ \\
Dissimilarity & 0.199 & -0.123 & -0.278 & -0.142 & 0.383 \\
Elevation & -0.914 & -0.055 & 0.274 & 0.232 & -0.048 \\
Eigenvalue & 6.221 & 4.883 & 3.638 & 2.882 & 1.516 \\
Percentage of variance (\%) & 29.623 & 23.253 & 17.322 & 13.723 & 7.217 \\
Cumulative percentage of variance (\%) & 29.623 & 52.876 & 70.198 & 83.921 & 91.138 \\
\hline
\end{tabular}

Note: We retained all principal components with eigenvalues greater than 1 . Loadings occupied first three are given in bold for each component. PC1 mainly corresponds to temperatures, while PC2 mainly corresponds to precipitation.

Table 2 The parameters and relative contributions of the environmental variables in Maxent model.

\begin{tabular}{lllllllllll}
\hline Clade & Parameter & AUC & bio1 & bio2 & bio3 & bio7 & bio8 & bio12 & bio14 & bio15 \\
\hline E. A & LQ1 & 0.979 & $\mathbf{0 . 2 3 1}$ & 0.033 & 0.12 & 0 & 0 & 0.163 & $0.301^{*}$ & 0.153 \\
E. B & LQ1 & 0.977 & 0.012 & 0.313 & 0 & 0.026 & 0 & $\mathbf{0 . 0 9 7}$ & $0.308^{*}$ & 0.172 \\
E. C & LQ2 & 0.937 & 0.194 & 0 & $\mathbf{0 . 3 6 8}$ & 0.001 & 0.006 & $0.255^{*}$ & 0.174 & 0.001 \\
E. D & LQ3 & 0.99 & 0.244 & 0 & $\mathbf{0 . 3 7 3}$ & 0.01 & 0 & $0.254^{*}$ & 0.119 & 0 \\
W. a & L2.5 & 0.892 & 0.062 & 0 & $\mathbf{0 . 7 0 3}$ & 0.029 & 0.026 & 0 & $0.174^{*}$ & 0.002 \\
W. b & LQH4 & 0.914 & $\mathbf{0 . 6 5 2}$ & 0 & 0.184 & 0 & 0 & 0.115 & 0.049 & 0 \\
\hline
\end{tabular}

Note: 1. Abbreviations: $\mathrm{L}=$ linear, $\mathrm{Q}=$ quadratic, $\mathrm{H}=$ hinge.

2. The environmental variables with highest gain when used in isolation are given in bold for each clade; the variables that decrease the gain the most when they are omitted are given * for each clade.

Table 3 Niche changes indices between native range and invaded clades.

\begin{tabular}{lllllll}
\hline Clades & Native clades & D & Sim. test & E & S & U \\
\hline E. A & E. B & 0.252 & 0.881 & $\mathbf{0 . 6 1 0}$ & 0.390 & 0.603 \\
E. A & E. C & 0.220 & 0.980 & 0.485 & $\mathbf{0 . 5 1 5}$ & 0.302 \\
E. B & E. C & 0.110 & 0.940 & $\mathbf{0 . 7 0 0}$ & 0.300 & 0.611 \\
E. A & E. D & 0.001 & 0.743 & $\mathbf{0 . 9 9 7}$ & 0.003 & 0.986 \\
E. B & E. D & 0.011 & 0.812 & $\mathbf{0 . 9 5 6}$ & 0.044 & 0.744 \\
E. C & E. D & 0.095 & 0.842 & $\mathbf{0 . 9 0 7}$ & 0.093 & 0.656 \\
E. A & W. a & 0.002 & 0.792 & $\mathbf{0 . 9 9 0}$ & 0.010 & 0.979 \\
E. B & W. a & 0.002 & 0.782 & $\mathbf{0 . 9 8 6}$ & 0.014 & 0.970 \\
E. C & W. a & 0.065 & 0.782 & $\mathbf{0 . 8 9 4}$ & 0.106 & 0.838 \\
E. D & W. a & 0.459 & $\mathbf{0 . 0 1 0}$ & 0.159 & $\mathbf{0 . 8 4 1}$ & 0.289 \\
E. A & W. b & 0.070 & 0.822 & 0.715 & 0.285 & $\mathbf{0 . 7 5 2}$ \\
E. B & W. b & 0.017 & 0.683 & $\mathbf{0 . 9 1 3}$ & 0.087 & 0.899
\end{tabular}




\begin{tabular}{lllllll}
\hline Clades & Native clades & D & Sim. test & E & S & U \\
\hline E. C & W. b & 0.307 & $\mathbf{0 . 0 5 0}$ & 0.304 & $\mathbf{0 . 6 9 6}$ & 0.493 \\
E. D & W. b & 0.444 & $\mathbf{0 . 0 2 0}$ & 0.037 & $\mathbf{0 . 9 6 3}$ & 0.628 \\
W. a & W. b & 0.527 & $\mathbf{0 . 0 1 0}$ & 0.031 & $\mathbf{0 . 9 6 9}$ & 0.514 \\
\hline
\end{tabular}

Note: Abbreviations: D: Schoener's D: the overlap value between pairwise clades; Sim. test: the p-value of the niche similarity test; E: niche expansion; S: stability; U: unfilling. Boldface values indicate significant similarity with $\mathrm{p}<0.05$ and the preferred niche shift model.

Table 4 Phylogenetic signal and model fitting analyses obtained for five climate principal components and Elevation.

\begin{tabular}{|c|c|c|c|c|c|c|c|c|c|c|c|}
\hline & & & $\mathrm{BM}$ & & & $\mathrm{OU}$ & & & EB & & \\
\hline & K (P - value $)$ & $-\ln L$ & $\mathrm{AICc}$ & $\omega$ & $-\ln L$ & AICc & $\omega$ & $-\ln L$ & AICc & $\omega$ & $-\ln L$ \\
\hline PC1 & $0.950(0.21)$ & -9.744 & 27.488 & 0.428 & -9.719 & 7.438 & 0.003 & -9.744 & & 0.003 & 465 \\
\hline PC2 & $0.749(0.33)$ & -12.033 & 32.066 & 0.353 & -12.009 & 42.017 & 0.002 & -12.033 & 42.066 & 0.002 & -11.434 \\
\hline PC3 & $1.141(0.09)$ & -9.781 & 27.561 & 0.627 & -9.780 & 37.559 & 0.004 & -9.781 & 37.561 & 0.004 & -10.324 \\
\hline PC4 & $1.648(0.04)$ & -9.266 & 26.532 & 0.665 & -8.941 & 35.881 & 0.006 & -9.262 & 36.523 & 0.005 & -9.986 \\
\hline PC5 & $1.381(0.049)$ & -4.858 & 17.716 & 0.572 & -4.858 & 27.716 & 0.004 & -4.847 & 27.693 & 0.004 & -5.166 \\
\hline Elev. & $1.568(0.045)$ & -45.06 & 98.121 & 0.687 & -45.06 & 108.121 & 0.005 & -45.06 & 108.121 & 0.005 & -45.877 \\
\hline
\end{tabular}

Note: Abbreviations: K: Blomberg's K; BM: Brownian motion; EB: Early burst; WN: White noise; OU: Ornstein-Uhlenbeck with one adaptative peak; - ln L: values for Neperian logarithm; AICc: corrected Akaike information criterion; $\omega$ : Akaike weights. Elev. = Elevation. Boldface values indicate the preferred model.

Table 5 The results of phylogenetic signal test and model fitting analyses based on size-corrected characteristic data.

\begin{tabular}{|c|c|c|c|c|c|c|c|c|c|c|c|c|}
\hline & K (P-value) & & $\mathrm{BM}$ & & & OU & & & EB & & & \\
\hline & & & AICc & $\omega$ & & AICc & $\omega$ & $-\ln L$ & $\mathrm{AICc}$ & $\omega$ & & \\
\hline SVL & $1.222(0.07)$ & & & 17 & & & 0.004 & & & .004 & & \\
\hline HL & & 75 & 7.149 & .443 & 7.648 & -17.296 & 0.003 & 7.575 & 7.149 & .003 & 7.791 & \\
\hline HW & 0 & 23 & 6.646 & 0.4 & 0 & 21 & 0 & & & 003 & 678 & \\
\hline SL & 1 & 18 & 37 & 0.5 & 1 & -29 . & 0.004 & 18 & & .003 & & \\
\hline INS & 1 & & -40.8 & & & & 0.0 & & & 0.004 & & \\
\hline IOS & 0.8 & 1.957 & -35.914 & 0.3 & 2.049 & -26.098 & 0.003 & 21. & & 0.002 & 536 & \\
\hline UEW & 0 & & & & & & 0.002 & & & 0.002 & 959 & \\
\hline ED & ) & 1 & -36.5 & 0.31 & 22.409 & -26.819 & 0.002 & 22.291 & -26 . & 0.002 & 083 & \\
\hline LAHL & & 25 & & 0.2 & 17.013 & -16.026 & 0.002 & 16. & -15 & 0.002 & 787 & \\
\hline LAD & 0.502 & .182 & & 0.189 & 20.305 & -22.610 & 0.001 & 20.182 & -22.364 & 0.001 & 21.634 & \\
\hline HLL & 0.565( & & & 0.25 & 13.899 & -9.7 & 0.002 & 13.808 & -9.616 & 0.002 & 14.662 & \\
\hline TL & $0.359(0.9$ & 9.975 & & 0.096 & 20.180 & -22.359 & 0.001 & 19.975 & -21.951 & 0.001 & 22.220 & \\
\hline TW & $0.376(0$ & & & 0.092 & 8697 & 0.6 & 0.001 & & 1.027 & 0.001 & 10.773 & \\
\hline TFL & & & & & & & 0.002 & & -7.964 & 0.002 & 14.115 & \\
\hline FL & $0.587(0.65)$ & 16.235 & -24.470 & 0.288 & 16.324 & -14.648 & 0.002 & 16.238 & -14.476 & 0.002 & 17.132 & \\
\hline
\end{tabular}

Note: Abbreviations: K: Blomberg's K; BM: Brownian motion; EB: Early burst; WN: White noise; OU: Ornstein-Uhlenbeck with one adaptative peak; - ln L: values for Neperian logarithm; AICc: corrected Akaike 
information criterion; $\omega$ : Akaike weights. Boldface values indicate the preferred model.

Table 6 Model comparison based on AICc value between climate principal components and traits.

\begin{tabular}{|c|c|c|c|c|c|c|c|c|c|}
\hline \multirow[t]{2}{*}{ Model } & \multirow[b]{2}{*}{ SVL } & \multirow[b]{2}{*}{ LAD } & \multirow[b]{2}{*}{ HW } & \multirow[b]{2}{*}{ SL } & \multirow[b]{2}{*}{ LAHL } & \multirow[b]{2}{*}{ TFL } & \multicolumn{3}{|c|}{ AICc } \\
\hline & & & & & & & FL & HL & HLL \\
\hline PC1 & 40.54 & -32.91 & -23.63 & 14.46 & -18.89 & -10.57 & -16.26 & -23.58 & -11.4 \\
\hline $\mathrm{PC} 2$ & 48.42 & -26.64 & -17.77 & 13.55 & -18.93 & -10.25 & -17.31 & -17.65 & -13 \\
\hline PC3 & 37.16 & -27.12 & -24.63 & 13.63 & -17.82 & -10.55 & -16.8 & -22.37 & -12.9 \\
\hline $\mathrm{PC} 4$ & 44.26 & -25.48 & -20.55 & 12.84 & -19.76 & -11.25 & -16.36 & -23.03 & -11.7 \\
\hline PC5 & 48.33 & -26.09 & -17.46 & 14.11 & 48.33 & -10.73 & -17.13 & -18.54 & -11.4 \\
\hline $\mathrm{PC} 1+\mathrm{PC} 2$ & 65.89 & -4.16 & 1.84 & 43.32 & 10.15 & 19.38 & 12.46 & 6.04 & 16.76 \\
\hline $\mathrm{PC} 1+\mathrm{PC} 3$ & 66.02 & -7.3 & 4.61 & 42.38 & 10.16 & 19.41 & 12.47 & 6.11 & 13.16 \\
\hline $\mathrm{PC} 1+\mathrm{PC} 4$ & 65.82 & -14.08 & 4.38 & 42.67 & 9.83 & 18.72 & 13.5 & 1.01 & 18.28 \\
\hline PC1+PC5 & 70.01 & -6.75 & 6.36 & 44.08 & 9.02 & 18.97 & 12.8 & 4.39 & 18.57 \\
\hline $\mathrm{PC} 2+\mathrm{PC} 3$ & 66.98 & -0.39 & 5.36 & 42.97 & 10.41 & 19.44 & 12.37 & 6.9 & 15.88 \\
\hline $\mathrm{PC} 2+\mathrm{PC} 4$ & 73.99 & 3.33 & 8.95 & 41.64 & 73.99 & 18.75 & 12.52 & 6.92 & 16.64 \\
\hline $\mathrm{PC} 2+\mathrm{PC} 5$ & 77.93 & 1.21 & 12.05 & 42.24 & 9.86 & 19.15 & 12.23 & 11.39 & $16.5 \varepsilon$ \\
\hline $\mathrm{PC} 3+\mathrm{PC} 4$ & 64.83 & 1.06 & 4.66 & 42.76 & 9.96 & 18.75 & 12.07 & 4.73 & 17.07 \\
\hline $\mathrm{PC} 3+\mathrm{PC} 5$ & 66.64 & 1.69 & 5.37 & 43.3 & 10.39 & 19.01 & 12 & 6.28 & 17.1 \\
\hline $\mathrm{PC} 4+\mathrm{PC} 5$ & 65.24 & 3.28 & -2.41 & 42.38 & 10.09 & 18.73 & 12.4 & 3.98 & 17.97 \\
\hline $\mathrm{PC} 2+\mathrm{PC} 3+\mathrm{PC} 4+\mathrm{PC} 5$ & $-103.42^{*}$ & $-159.79 *$ & -143.09 & -78.36 & -113.52 & -101.33 & -108.7 & -121.5 & -106. \\
\hline $\mathrm{PC} 1+\mathrm{PC} 3+\mathrm{PC} 4+\mathrm{PC} 5$ & -84.8 & -143.73 & $-151.84^{*}$ & $-78.8^{*}$ & -114.63 & -101.44 & -109.15 & -122.99 & -108 . \\
\hline $\mathrm{PC} 1+\mathrm{PC} 2+\mathrm{PC} 4+\mathrm{PC} 5$ & -83.85 & -149.04 & -141.71 & -78.57 & -112.79 & -101.3 & -108.14 & -121.38 & -105 . \\
\hline $\mathrm{PC} 1+\mathrm{PC} 2+\mathrm{PC} 3+\mathrm{PC} 5$ & -70.04 & -147.83 & -138 & -78.14 & -116.42 & -102.34 & -109.78 & -127.62 & -109 \\
\hline $\mathrm{PC} 1+\mathrm{PC} 2+\mathrm{PC} 3+\mathrm{PC} 4$ & -74.42 & -143.58 & -144.88 & -78.5 & -115.07 & -101.8 & -109.16 & -124.89 & -108 . \\
\hline
\end{tabular}

Note: Boldface values indicate the preferred model; * Significant difference with $\mathrm{p}<0.05$.

\section{Hosted file}

Figures and legends.pdf available at https://authorea.com/users/400321/articles/512661climatic-niche-evolution-with-key-morphological-innovations-across-clades-withinscutiger-boulengeri-anura-megophryidae 